\title{
Recoverable Effects Related to Pseudoelastic Cycling in Cu-Zn-Al Single Crystals
}

\author{
A. Yawny, J. Malarría*, F.C. Lovey and M. Sade \\ Comisión Nacional de Energía Atómica, Centro Atómico Bariloche, 8400 Bariloche, Argentina \\ * Consejo Nacional de Investigaciones Científicas y Técnicas (CONICET), Argentina
}

\begin{abstract}
Pseudoelastic cycling of $\mathrm{Cu}-\mathrm{Zn}-\mathrm{Al}$ single crystals has been shown to induce microstructural changes in the material. The mechanical response is also altered. However, some of the observed changes in the mechanical behavior are at least partially recovered at temperatures at which point defects are mobile. In this work results are presented in order to analyze the different contributions associated to the changes of the mechanical behavior during pseudoelastic cycling and in a subsequent ageing stage in the $\beta$-phase condition for $\mathrm{Cu}-\mathrm{Zn}-\mathrm{Al}$ single crystals. The magnitudes and kinetics of the recoverable changes are analyzed and compared with the other known phenomena that take place at these temperatures, i.e. the martensite stabilization and the beta phase recovery. Results show that the evolution is not simply related to these phenomena. Additional contributions, like point defects generation during cycling and the effect of dislocation array on the beta phase recovery, need to be considered.
\end{abstract}

\section{INTRODUCTION}

Fatigue mechanisms associated with the cycling of shape memory alloys through a martensitic transformation are a problem of academic and technological interest [1-4]. A repeated pseudoelastic transformation induces different microstructural changes [5-7] and can finally lead to the fracture of the sample. These changes are related to the nucleation and growth of bulk and surface defects $[2,8]$. The surface defects, aligned parallel to the habit plane of the transformation (HP-defects) with an intrusionextrusion morphology, are the main source of crack nucleation and form after cycling at temperatures above approximately $173 \mathrm{~K}$ [2]. The HP defects production is sharply reduced below this temperature level, giving rise to a longer fatigue life of the material [9].

Moreover, the change in the mechanical behavior observed in pseudoelastic cycling experiments has been explained by the formation of bulk defects, i.e. dislocations bands with retained martensite aligned parallel to the basal plane of the martensite (BP-defects). The variation of the hysteresis and slope of the pseudoelastic cycle in fatigue experiments depend strongly on the temperature in good correspondence with the type and density of defects [10].

At the liquid nitrogen temperature, the stress-strain curves show striking changes from the early stage of cycling, characterized by a gradual hardening process and widening of the hysteresis loop, until a saturation stage is reached [11]. These changes have been found to be partially recovered after ageing at room temperature. These phenomena have been explained considering that point defects are introduced during cycling at low temperatures and that they move above $273 \mathrm{~K}$ in these alloys.

Pseudoelastic fatigue cycling performed at around room temperature, shows less important changes in the stress strain curves and correspondingly a rather low BP-defect density [10]. The evolution of mechanical curves is mainly characterized by a gradual reduction of the critical stress $\tau^{\beta \rightarrow m}$ necessary to start the transformation [7]. The slope associated to the beta-martensite transformation is then correspondingly increased [2]. The microstructure shows a different picture, characterized by dislocation bands lying parallel to the habit plane of the induced martensite, and by a very low BP-defect density [7]. 
When analyzing the behavior around room temperature, the occurrence of diffusional processes should be taken into account [12]. In fact, the stabilization of the martensite has been considered to be the main cause of the decrease in the stress of transformation $\tau^{\beta \rightarrow m}$ [7]. Additionally, strong recovery effects in the lowered critical stress $\tau^{\beta \rightarrow m}$ and in the slope have beeen observed after cycling by ageing in the betaphase at the temperature of the experiment [11].

The aim of this work is to analyze the contribution of diffusional processes on the recovery of the effects introduced during pseudoelastic cycling, in a temperature range in which diffusional mechanisms are important.

\section{EXPERIMENTAL DETALS}

Single-crystals of beta phase $\mathrm{Cu}-\mathrm{Zn}-\mathrm{Al}$ alloys with electronic concentration $\mathrm{e} / \mathrm{a}=1.48$ were grown by the Bridgman method in sealed vycor glass. From them, tensile cylindrical samples of $3 \mathrm{~mm}$ diameter were spark machined with gauge length $\mathbf{l}_{\mathbf{o}}$ indicated in Table I. The tensile axis orientation was determined by the X-ray Laue technique. The Schmid factors $\mu$ for the most favorable tensile stress induced variant of the $18 \mathrm{R}$ martensite are shown in Table I. Samples A to $\mathrm{G}$ in Table I were annealed at $1123 \mathrm{~K}$ for 20 minutes and air cooled to room temperature; afterwards they were kept at least for 4 days at this temperature. After treating the surface by mechanical and electrolytic polishing, the samples were kept at the test temperature for a time interval $t_{1}$ indicated in Table I. Finally the samples A, B, C, D, E, F and G were cyclically strained under tensile stress at constant temperature $\mathbf{T}_{\text {exp }}$ between a purely $\boldsymbol{\beta}$-phase state (at approximately half the elastic range) and a $100 \%$ martensite transformed state, except sample $\mathrm{A}$, which was transformed only up to $50 \%$ martensite during each cycle. A number of cycles $\mathbf{N}$ were performed in this way at a frequency $v$, without interruption. The maximum applied stress $\tau_{M}$ during cycling is shown in Table I. After the cycling was stopped the evolution of the stress-strain behavior was analyzed by performing a pseudoelastic cycle and determining the stress $\tau^{\beta \rightarrow m}$ to induce the transformation. This procedure was repeated after several time intervals. In this way the evolution of $\tau^{\beta \rightarrow m}$ with the ageing time was obtained and the kinetics of the $\beta$-phase recovery process characterized. This stage of the experiment will be named from now on the $\beta$-phase recovery process. The crosshead speed used for this analysis was $0.1 \mathrm{~mm} / \mathrm{min}$, an elongation rate sufficiently low to disregard temperature effects on the hysteresis of the cycles. A complementary experiment was performed with sample $H$. The thermal treatment for this sample was 20 minutes at $1123 \mathrm{~K}$, air cooling to $353 \mathrm{~K}$, two days at this temperature and finally a static stabilization process which will be explained below.

\section{EXPERIMENTAL RESULTS}

The evolution of the recovery process may be followed by the measurement of different parameters. In this work two of them were considered: the slope associated to the transformation and the critical stress $\tau^{\beta \rightarrow m}$ to start the transformation. In samples $A$ to $G$ a decrease of $\tau^{\beta \rightarrow m}$ and an increase of the mentioned slope were found.

As an example in Fig. la the change in the pseudoelastic stress-strain behavior upon cycling of the sample $\mathrm{G}$ can be appreciated. After a number of cycles $\mathrm{N}=5750$ the $\tau^{\beta \rightarrow m}$ stress diminished in $6.2 \mathrm{MPa}$. The transformation slope clearly increased. In Fig. Ib the final recovered cycle, after a total ageing time of $117828 \mathrm{~s}$, is plotted in comparison with cycle $N$. The recovered critical stress $\tau^{\beta \rightarrow m}$ and slope reached values close to those before the fatigue cycling.

Fig. 1 shows that the whole hysteresis cycle in the recovered stage $(\mathrm{N}=5759)$ slightly shifted towards lower values of stresses. Such shifts did not show a systematic behavior for all the studied specimens. Isalgue et. al. [15] have shown that the $\mathrm{M}_{s}$ of the specimen is a function of the temperature and 
time in the range of temperatures where diffusional process are possible. Thus, the shift observed between the initial cycle and the recovered one is attributed to the adjustment of the $M_{s}$ to its corresponding value at the working temperature, which depends strongly on the thermal history of the specimen, the temperature, the time and the cycling procedure.

Table I: Sample characteristics and experimental parameters. Ms is the measured transformation temperature, $\mu$ the orientation of the most favorable stress induced variant (Schmid factor), $\mathbf{I}_{\mathbf{0}}$ the sample length, $\mathbf{t}_{\mathbf{1}}$ the time at the experimental temperature $\mathbf{T}_{\text {exp }}$ previous to the fatigue cycling, $\boldsymbol{v}$ the cycling frequency, $\mathbf{N}$ the total number of cycles previous to the $\beta$-phase ageing stage, $\tau^{\beta \rightarrow m}$ the resolved shear stress for beta to martensite transformation previous to the fatigue cycling, $\tau_{M}$ the maximum resolved shear stress in the whole experiment, $t_{2}$ a rough estimate of the time constant of the $\beta$-phase recovery. The composition of the alloys in atomic percent is : $14.944 \mathrm{Zn}, 16.528 \mathrm{Al}$, Cu rest, for samples $\mathrm{A}, \mathrm{B}, \mathrm{C}, \mathrm{D}$ and $14.151 \mathrm{Zn}, 16.925$ Al for sample $E, G, H$.

\begin{tabular}{|c|c|c|c|c|c|c|c|c|c|c|}
\hline SAMPLE & $\begin{array}{c}\text { Ms } \\
(\mathbf{K})\end{array}$ & $\boldsymbol{\mu}$ & $\begin{array}{c}\mathbf{l o} \\
(\mathbf{m m})\end{array}$ & $\begin{array}{c}\mathbf{t}_{1} \\
(\mathbf{h})\end{array}$ & $\begin{array}{c}\mathbf{T e x p} \\
(\mathbf{K})\end{array}$ & $\begin{array}{c}\mathbf{v} \\
(\mathbf{H z})\end{array}$ & $\mathbf{N}$ & $\begin{array}{c}\tau^{\beta \rightarrow m} \\
(\mathrm{MPa})\end{array}$ & $\begin{array}{c}\tau_{\mathbf{M}} \\
(\mathbf{M P a})\end{array}$ & $\begin{array}{c}\mathbf{t}_{2} \\
(\mathbf{s})\end{array}$ \\
\hline $\mathbf{A}$ & 267 & 0.44 & 17 & 24 & 301 & 0.11 & 15200 & 34.8 & 38 & 34400 \\
\hline $\mathbf{B}$ & 279 & 0.33 & 10 & 6 & 306 & 0.15 & 5160 & 27.6 & 29.0 & - \\
\hline $\mathbf{C}$ & 280 & 0.48 & 21 & 17 & 307 & 0.06 & 3553 & 28.1 & 42.4 & 8105 \\
\hline $\mathbf{D}$ & 276 & 0.43 & 10 & 26 & 315 & 0.006 & 2011 & 38.9 & - & - \\
\hline $\mathbf{E}$ & 296 & 0.38 & 10 & 72 & 318 & 0.12 & 8390 & 22.5 & 23.7 & 9987 \\
\hline $\mathbf{F}$ & 284 & 0.49 & 10 & 6 & 324 & 0.8 & 10000 & 40.7 & 48.0 & 5723 \\
\hline $\mathbf{G}$ & 302 & 0.38 & 10 & 72 & 357 & 0.1 & 5760 & 54.9 & 55.2 & 4952 \\
\hline $\mathbf{H}$ & 293 & 0.33 & 10 & 48 & 347 & - & - & 43.1 & 44.9 & - \\
\hline
\end{tabular}

Figure 2 shows the critical stress to transform $\tau^{\beta \rightarrow m}$ as a function of the time in the recovery stage for several samples that were previously cycled at different temperatures. The $\tau^{\beta \rightarrow m}$ level before cycling is indicated in dashed horizontal lines as a reference. We notice a clear evolution which leads to a partial or total recovery of this stress. A similar time evolution is obtained for each sample, if the slope associated to the pseudoelastic transformation is considered instead of $\tau^{\beta \rightarrow m}$. A rough estimation of the time constant $\left(t_{2}\right)$ for each case is displayed in Table $I$.

Fig. 3 presents the result of an experiment performed with sample $\mathrm{H}$ at $353 \mathrm{~K}$ to compare the beta recovery process after an static stabilization. The procedure was as follows: after two days at this temperature a first complete pseudoelastic transformation was performed (path $a b c d a$ ) and subsequently a partial transformation was induced until point $e$. At this point the strain was kept constant. The stress dropped from point $e$ to $f$ after $95 \mathrm{~min}$. After that the forward transformation was completed (path $f g h$ ) and finally the sample was unloaded. The stabilization of the partially transformed martensite is clearly observed along the path ijka. A beta recovery of about $80 \%$ was obtained after another complete cycle performed 80 seconds later (path amna). This time constant is by two orders of magnitude shorter than that shown in the Table I.

\section{DISCUSSION}

The results obtained in this work suggest the existence of recovering mechanisms at around roomtemperature that act to reduce some of the effects which are a consequence of the pseudoelastic cycling. The main effects, i.e., the gradual reduction of the critical stress $\tau^{\beta \rightarrow m}$ necessary to start the transformation and the increase in the slope associated to the beta-martensite transformation, are partially or completely recovered by ageing in the $\beta$-phase after cycling. It is interesting to compare the present results with those 
corresponding to recently published tests performed at lower temperatures. Malarría et al. found that the hysteresis and the slope associated to the transformation in the stress-strain behavior strongly increase during cycling, leading to a strong hardening $[10,11]$. No recovery processes were observed by holding the samples at low temperature, but part of the slope was recovered by heating the material up to temperatures close to or above room temperature. The introduction of point defects were postulated to explain this effect [11]. Tolley et al. showed that vacancies are mobile at $\mathrm{T}>273 \mathrm{~K}$ for these alloys [13], explaining the observed recovery. However there is an important difference between the experiments mentioned above and those presented here. In the present case, diffusion in the $\beta$-phase and in the martensite are present during the whole cycling procedure.

The decrease of $\tau^{\beta \rightarrow m}$ observed in Fig. 1a is probably associated to a stabilization of the martensite followed by an incomplete $\beta$ recovery in each cycle. Since each area of the specimen remains a different time in the martensite state the increasing slope can also be explained in this way. However, the longer time constant observed for the $\beta$-phase recovery does not match with that observed after normal stabilization process [14]. The difference could be related to the presence of dense dislocation arrays, which are also produced during cycling $[8,10]$.

According to the results of recent published works $[15,16]$ the stabilization of martensite is enhanced in the presence of a moving interface, i.e., during cycling. On the other hand, the recovery of the $\beta$-phase occurs when only one phase is present in the material; in this case the dislocations might serve as sinks for the point defects giving rise to a slower diffusional mechanism for this recovery.
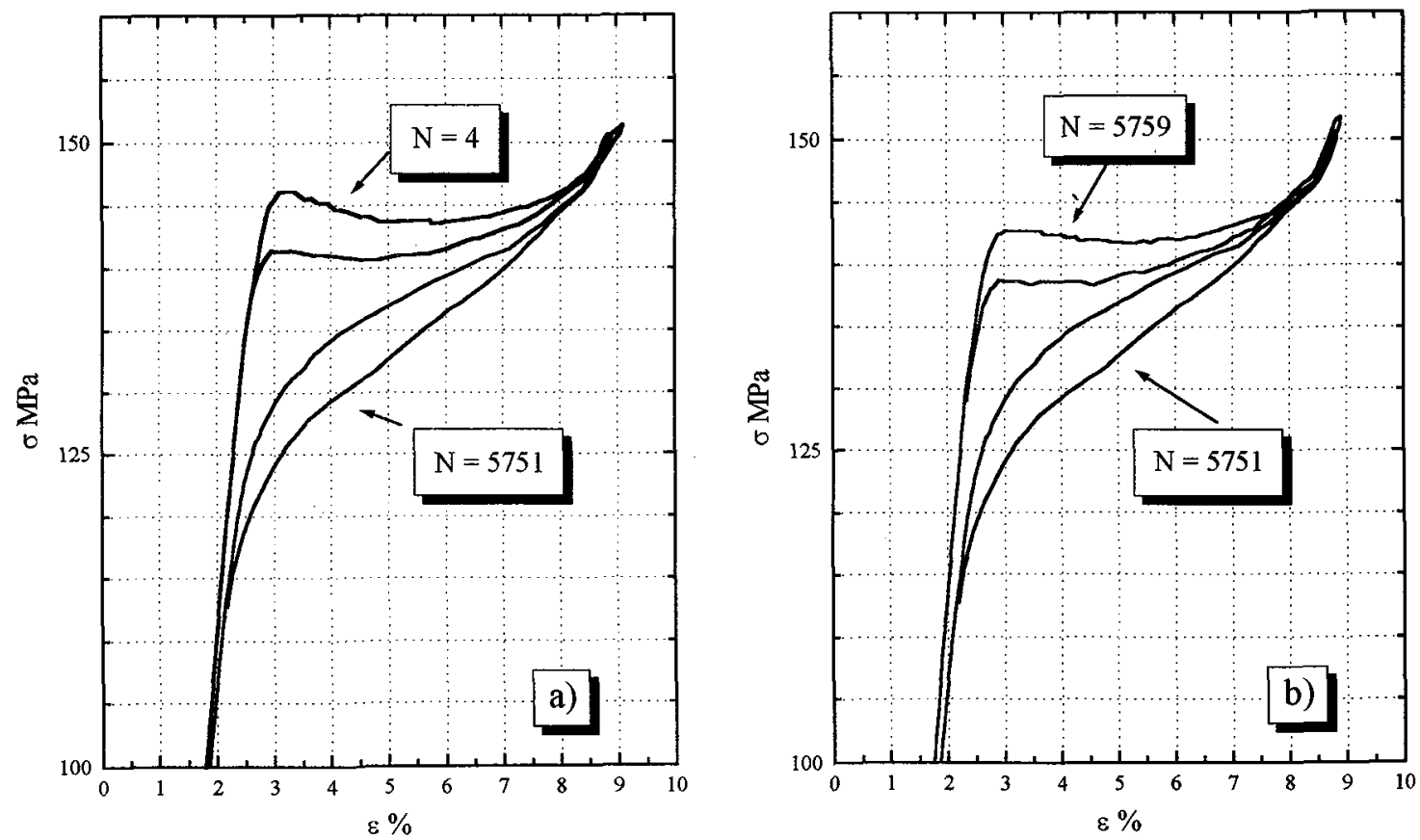

Figure 1: Pseudoelastic cycles. a) Cycles $\mathrm{N}=4$ at the start of the fatigue experiment and $\mathrm{N}=5751$ immediately after the continuos cycling. b) $\beta$ phase recovery: cycle $\mathrm{N}=5751$ and cycle $\mathrm{N}=5759$ obtained 33 hs. after ageing started. Test temperature $=357 \mathrm{~K}$.

$\sigma=$ tensile applied stress, $\varepsilon=$ deformation of the sample. 


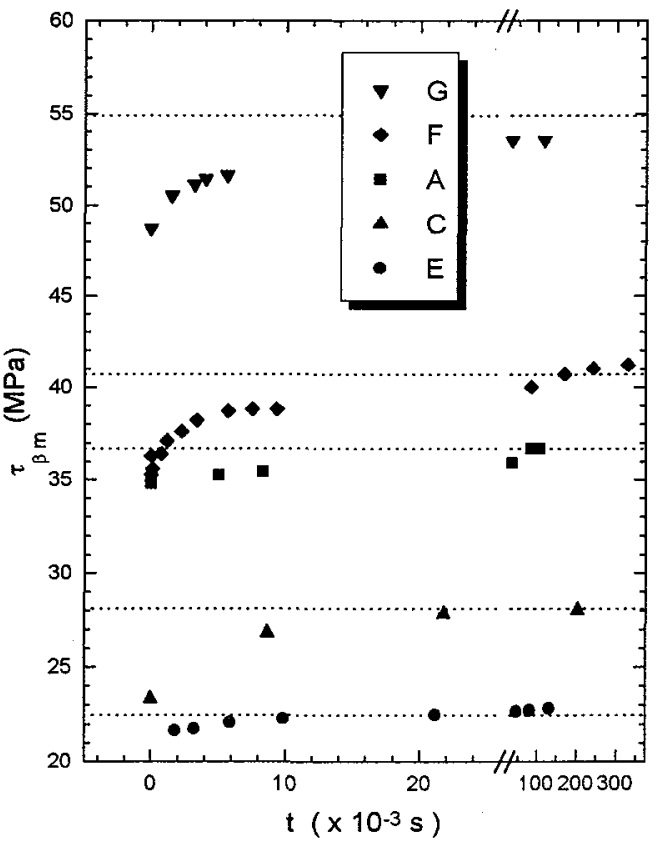

Figure 2: Critical resolved shear stress to start the transformation $\tau^{\beta \rightarrow m}$ vs. elapsed time in the $\beta$ phase recovery stage. Horizontal dashed lines indicate the $\tau^{\beta \rightarrow m}$ previous to cycling.

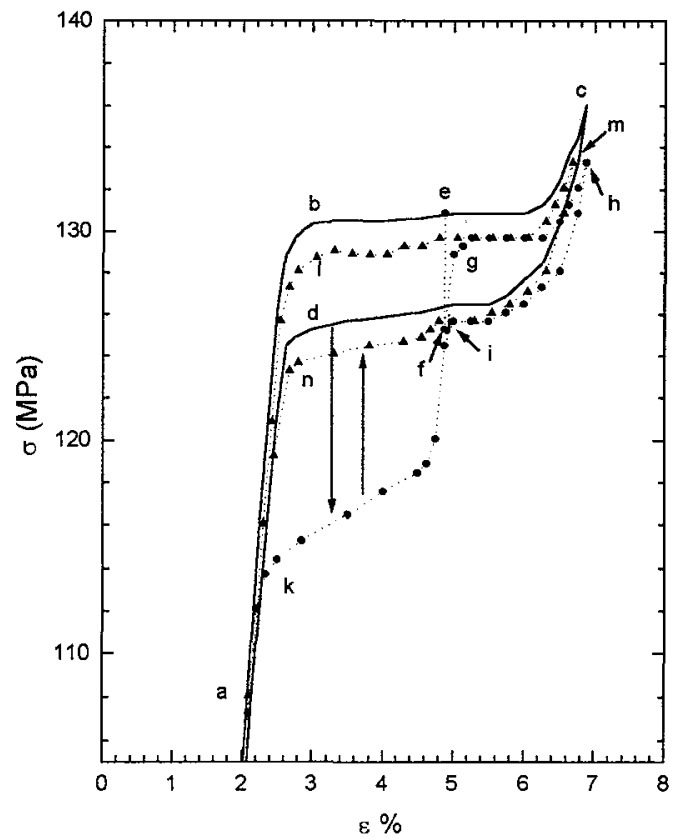

Figure 3: Stabilization and $\beta$-phase recovery experiment with sample $\mathbf{H}$, at $348 \mathrm{~K}$. abcda: reference complete cycle before stabilization (full line); abe: second partial transformation. At point $e$ the elongation was stopped and kept constant during 95 minutes, the stress decreased from $e$ to $f$. The transformation was completed and the sample unloaded: fghijka. An immediately obtained cycle is indicated as almna. The amount of the stabilization effect and the following recovery are indicated by the vertical down and up arrows respectively.

\section{Acknowledgments}

Single crystals and samples were made by Mr. Carlos Gomez.

\section{References}

[1] Van Humbeeck J., Journal de Physique IV, Colloque C4, supplément au Journal de Physique III, Vol.1 (1991), C4-189.

[2] Sade M., Rapacioli R. and Ahlers M., Acta Metall. 33 (1985) pp. 487.

[3] Thumann M. and Hornbogen E., Z.Metallkde. 79 (1988) pp. 119.

[4] Hornbogen E., Z. Metallkd. 86 (1995) pp. 656.

[5] Lai M.O., Lu L. and Lee W.H., J. of Mat. Science 31 (1996) pp. 1537.

[6] Sade M. and Hornbogen E., Z.Metallkde. 79, (1988) 782.

[7] Malarría J., Sade M. and Lovey F., J. Phys. Colloq.C8, v.5 (1995) pp. C8-889. 
[8] Sade M., Uribarri A. and Lovey F., Philos. Mag. A, 55 (1987) pp. 445.

[9] Sade M. and Ahlers M., Scr. Metall. 19 (1985) pp. 425.

[10] Malarría J. and Sade M., Scripta Met. 30 (1994) pp. 241.

[11] Malarría J., Sade M. and Lovey F., Z. Metallkd. 87 (1996) pp. 953.

[12] Cingolani E., Yawny A. and Ahlers M., J. Phys. Colloq.C8, v.5 (1995) pp. C8-865.

[13] Tolley A. and Ahlers M., J. of Nucl. Mat. 205 (1993), pp.339.

[14]Abu Arab A. and Ahlers M., Acta Metall. 36 (1988) pp.2627.

[15] Isalgue A., Lovey F., Pelegrina J. and Torra V., J. de Physique IV, Colloque C8, supplément au Journal de Physique III, 5 (1995) pp. C8-853.

[16] Pelegrina J., Rodríguez de Rivera M., Torra V. and Lovey F., Acta Metall. Mater. 43 (1995) pp.993. 